\title{
WHAT IS THE MEANING OF SHARING: INFORMING, BEING INFORMED OR INFORMATION OVERLOAD?
}

An explorative study of implementing an Enterprise Social Media Platform in a public organization

by Halvdan Haugsbakken

In recent years, several Norwegian public organizations have introduced Enterprise Social Media Platforms. The rationale for their implementation pertains to a goal of improving internal communications and work processes in organizational life. Such objectives can be attained on the condition that employees adopt the platform and embrace the practice of sharing. Although sharing work on Enterprise Social Media Platforms can bring benefits, making sense of the practice of sharing constitutes a challenge. In this regard, the paper performs an analysis on a case whereby an Enterprise Social Media Platform was introduced in a Norwegian public organization. The analytical focus is on the challenges and experiences of making sense of the practice of sharing. The research results show that users faced challenges in making sense of sharing. The paper indicates that sharing is interpreted and performed as an informing practice, which results in an information overload problem and causes users to become disengaged. The study suggests a continued need for the application of theoretical lenses that emphasize interpretation and practice in the implementation of new digital technologies in organizations. 


\section{N}

NORDIC JOURNAL

of Science and Technology Studies

\section{Introduction}

In the last decade, many private and public organizations have started to take great interest in Enterprise Social Media Platforms (ESMPS) (Leonardi, Huysman, \& Steinfield 2013). ESMPS, a term that implies an expansion of Enterprise 2.0 (MCAfee 2006), refers to a platform used for internal communication in organizations. ESMPs contain a range of features that are used to share and organize information, such as tagging systems, user profiles, search engines, follower features, discussion boards, and group features. Known examples of ESMPs are Yammer and Facebook@ work. The platforms are assumed to bring a range of benefits for organizations and for the organization of work processes. These benefits can include enhancement of the quality of internal communications and workflows. A central practice related to the successful use of ESMPs is active engagement by users or employees through the sharing or co-creation of content, although the workplace principle is not always easy to put into practice.

Since the end of the 2000s, several large Norwegian private and public organizations have introduced ESMPs to their employees. The incentive for their acquisition is motivated by various goals. For example, they can reduce internal organizational barriers, enhance organizational communications, and cut down on time spent sending e-mails. In this way, one can attain a greater overview of organizational activities and the competencies of employees. In this regard, ESMPs are presented as a solution that can contribute to solving traditional management challenges that are faced daily by public organizations. In the wake of this development, discourses focusing on the importance of sharing in organizations emerge. Top and middle managers stress the sharing of work and engagement via ESMPs as means of bringing about organizational change and unity and

\section{Theoretical perspective}

Sharing has emerged as a significant social action performed by billions of social media users worldwide. In general, sharing brings with it a range of claimed unintended consequences (Merton 1936), and can be defined as a practice that originates in reconstituting dynamics and reciprocal relationships between the material properties of social media and social action. As such, it has affected the organization of social life. For example, what people share on social media draws media attention and is predicted by traditional media as having positive and negative effects on our well-being. The sharing of experiences can create community awareness on civic matters that are important to society, but also accusations of egocentric behavior. We also see that ongoing online socialization may lead to new mediated practices such as phubbing and digital detox. Phubbing is defined as the act of ignoring a person's surroundings through the use of a cellphone, which is deemed an impolite action. Digital detox is understood as a period win which a person stops using electronic connecting devices such as smart the use of digital technologies in work life. Surfing on the top of such management discourses is an emphasis that employees embrace a "sharing culture." Such developments substantiate the importance of analyzing the meaning of sharing through social constructionist research perspectives regarding the use of technology in organizations.

In 2012, a Norwegian County Authority decided to upgrade its intranet to become an ESMP, an effort initiated by the top management. The goal was to simplify the worksurface because the employees previously worked across separate forms of ICTS. A further objective was to transfer work practices from e-mail and local storage to the newly acquired platform by sharing. Although the technical implementation of the ESMP was successful, the top management found that employees were not sharing work as intended. By using a practice perspective on technology and the organizing of work and related research on Enterprise Social Media (Orlikowski 2000; Orlikowski \& Gash 1994), this paper questions how a group of employees working in the County Authority interpret the meaning of sharing and put it into practice through the ESMP. The use of a practice perspective indicates that employees face challenges in interpreting the meaning of sharing. Sharing is interpreted and performed as an informing practice, which results in an information overload problem and disengaged users. In order to tackle the research question, the paper is divided into different parts. The following section addresses the scholarly discussion upon which the study is based. Thereafter, the research strategies used to complete this study are outlined. The research findings are subsequently presented, before the research results are discussed in relation to the relevant research horizon. The final part concludes the paper.

phones and tablets. These indicate that the organization of communicative practices in the digital sphere can become unmanageable and chaotic. Although research on social media and sharing has proliferated, organization researchers have yet to fully frame the impact of sharing on organizational life.

In consequence, such dynamics call for the development of a research perspective that discusses the meaning of sharing in organizations by use of ESMPs, especially where sharing assumes a different role than that intended. This argument is valid for several reasons. Surprisingly, organizational scholars who study knowledge-sharing processes by use of knowledge management systems (for example) claim that what is actually shared by users on platforms for the sharing of work has yet to be adequately framed (Ardichvili, Page, \& Wentling 2003). In particular, a knowledge gap seems to exist regarding the formation of sharing processes and how this is related to emergent properties coming from the use of 


\section{$\$$}

NORDIC JOURNAL

of Science and Technology Studies

recursive technology in work processes (Kosonen 2009). Instead, the knowledge management research stream has examined predefined assumptions of sharing (Chen \& Hung 2010; Wasko \& Faraj 2005) and conditions that prevent the sharing of knowledge in virtual communities (Ardichvili 2008; Ardichvili, Maurer, Li, Went Ling, \& Stuedemann 2006; Ardichvili et al. 2003).

With the advent of ESMPs in organizations I argue that there is an urgent need to formulate and facilitate a new and much broader research agenda. This has been seen in organizational research, which has introduced new definitions of platforms and has criticized existing definitions of social media for their shortcomings. An example of a new definition is Enterprise Social Media, which is defined as: "web-based platforms that allow workers to (1) communicate messages with specific coworkers or broadcast messages to everyone in the organization; (2) explicitly indicate or implicitly reveal particular coworkers as communication partners; (3) post, edit, and sort text and files linked to themselves or others; and (4) view the messages, connections, text, and files communicated, posted, edited, and sorted by anyone else in the organization at any time of their choosing" (Leonardi et al. 2013: 2). This definition is a modified version of Kaplan and Haenlein's (2010: 61), who define social media as a "group of Internet-based applications that build on the ideological and technological foundations of Web 2.0, and that allow the creation and exchange of User Generated Content."

Current definitions of social media are criticized for failing to adequately explain organizational processes (Treem \& Leonardi 2012). To accommodate these limitations, we find an emerging body of research studying ESMPs that uses a social constructionist research lens on technology and the organization of work (Leonardi \& Barley 2010). This research stream has (among others) developed an affordance perspective (Gibson 1986) to grasp the impact of ESMPs in organizations. Affordance (Gibson 1986) stresses the advantages of technologies. It argues that technologies can be perceived as beneficial in performing activities without paying attention to what an object "is," that is, to ask what it can afford (Treem \& Leonardi 2012). The focus on perception means to put emphasis on an object's utility, and affordance provides the possibility of understanding action potential and the capabilities of a technology, and how it can be linked to processes in organizations (Treem \& Leonardi 2012). The affordance lens is used to place greater emphasis on the meaning of materiality, which is said to have diminished in value as other concepts dominate the research agenda, such as sociomateriality (Mutch 2013). Affordance is linked to critical theory, which is deemed as providing new innovative ways of addressing the relationships between materiality and immateriality (Leonardi 2013). A critical theory approach assumes the existence of multiple realities that operate interchangeably and independently of one another, with the implication that actors and objects are self-contained entities that influence each other through impacts or social interaction (Leonardi 2013).
In contrast, sociomateriality (Orlikowski 2007), which draws on influences from Actor-Network-Theory (Callon 1986; Latour 1987. 2005), has emerged as an alternative sub-research stream to understanding the impact of social media in organizational studies. Sociomateriality, which assumes that "materiality is intrinsic to everyday activities and relations" (Orlikowski \& Scott 2008, 455), provides an alternative approach to understanding the meaning of technology and therefore what the potential consequences of social media might entail. Orlikowski argues that previous understandings of materiality in management studies were framed around an ontology of separateness (Suchman 2007), in which one theorized that the material and the immaterial are separate entities and realities (Orlikowski 2010). Sociomateriality instead argues that they should be seen as linked, equal, and inseparable, which means addressing a relational ontology. Therefore, future organizational researchers with an interest in social media could study technological artifacts "symmetrically to the humans, and as equivalent participants in a network of humans and non-humans that (temporarily) align to achieve particular effects" (Orlikowski 2010, 135). In this regard, Orlikowski and Scott (2014) apply a sociomaterial practice perspective in one of their latest research works on valuation regimes. They demonstrate that online evaluations of hotels performed by users on social media have drastic impact on the domestic travel industry (Orlikowski \& Scott 2014).

However, an important argument running through the above literature is the requirement for more theorizing. Current research focuses on a particular platform or features, leading to a claim that researchers are incapable of making inferences about the consequences of the material for organizing (Leonardi et al. 2013). Current definitions of ESMPs are too application-focused and overlook the social dynamics and reciprocal relationships between the material and the immaterial (Leonardi et al. 2013). Treem and Leonardi (2012) argue that this causes scholars to fail to possess sufficient terminology to explain the ways in which ESMPs can influence social behavior and to generalize matters to organizations across contexts. Here, the affordance lens offers researchers the possibility of making interesting analyses regarding the ways in which ESMPs influence organizational processes such as socialization and power aspects in organizations. Research has suggested various affordances that ESMPs can give for organizational processes. For example, Treem and Leonardi (2012) suggest that ESMPs can enable four affordances: visibility, edibility, persistence and association. A case in point highlighting the meaning of a singular affordance is Treem and Leonardi's (2012) argument that the affordance visibility is seen when employees use an ESMP to make their behavior, knowledge, preferences and commutation network visible to others. They argue that actions like posting updates, showing a list of friends and writing personal profiles are beneficial and enable the visualization of work to third parties. Leonardi $(2014,2015)$ illustrates this point by showing that work interaction on an ESMP platform is pivotal for knowledge work and for the transfer of knowledge in a large organization. Based on a study of a financial service in the United States of America, Leonardi shows 


\section{$\$$}

NORDIC JOURNAL

of Science and Technology Studies

that the use of a company's ESMP assists third-users to enhance awareness of meta knowledge, as one learns about the competencies of co-workers and the matters on which they are working. ESMPs can be used to make accurate interferences of people's meta knowledge and the sharing of co-workers' communication activities, and communicating via messenger software can offer innovative products and avoid the duplication of work. In a related study, Leonardi and Meyer (2015) develop the above claim in a study of a communications business unit in a telecommunication unit. Leonardi and Meyer test out a set of hypotheses and instances of knowledge transfer to show that when knowledge workers are exposed to communication activities on an ESMP, internal communication can be enhanced.

Beyond these works, researchers have theorized affordance in two other principle directions. First, we can identify works that conceive of affordance at a conceptual level. Second, researchers develop the term empirically through case study designs. An example of the former is a study by Majchrzak, Faraj, Kane, and Azad (2013), who demonstrate how ESMPs have four affordances to inspire engagement of visible knowledge conversations in organizations. These include met voicing, triggered attending, network-informed associating and generative role-taking. For example, met voicing would mean that an ESMP has the action capability to enable users to react to others' presence, profiles, content and activities. Ellison, Gibbs, and Weber (2015) develop a collective affordance and affordances for organizing, and explore the role of organizational affordances in light of the fact that organizations become distributed entities. The affordance lens needs to be broadened beyond the context of individual uses, which has been seen in many analyses. Ellison et al. (2015) note that the affordances of ESMPs can include concepts such as social capital dynamics, identity formation, context collapse and networked organizational structures. Fulk and Yuan (2013) argue that ESMPs have the affordance to solve organizational challenges and represent a preferable platform for organizing knowledge sharing in comparison to older knowledge management systems. Fulk and Yan argue that by combining transitive memory theory, public good theory and social capital theory, ESMPs have the affordance to deal with three associated challenges in the sharing of organizational knowledge. These include knowledge of the location of expertise in the organization, motivation to share knowledge, and the development and maintenance of relationships with knowledge providers. In considering work that uses the affordance lens for cases from organizational life, however, Vaast and Kaganer (2013) explore how organizations react to employees' adoption of ESMPs. Based on a sample of corporate policy documents, Vaast and Kaganer find that organizations view ESMPs as more of a risk than an asset. Oostervink, Agterberg and Huysman (2016) have undertaken a study connecting enactment to the affordance lens, as affordances are enacted in practice and institutional forces in an organization can shape how ESMPs are used by employees. Oostervink et al. point out that the institutional logics of a corporation and employees' professional expertise shape the knowledge that employees share on ESMPs. Although the affordance of visibility and associability are assumed to enhance knowledge sharing in organizations, Gibbs, Rozaidi and Eisenberg (2013) find the opposite effect. They performed a study among a group of engineers and noted that engaging EMPSs create contradictions in workplace interactions. For example, constantly remaining accessible and open to other suggests that one is a hassle, thus causing employees to feel that they need to hide certain behaviors from others. Based on this finding, Gibbs et al. (2013) have suggested that scholars theorize affordance in terms of dichotomies, not singular affordance concepts. They establish three affordances with which users interact when they use an ESMP. These include visibility-invisibility tension, engagement-disengagement tension, and sharing-control tension.

Organizational researchers have also explored ESMPs from other angles. Research shows that employees are receptive to ESMPs in certain organizations: those that make and sell the technology, being IT companies and organizations with the resources to research the technology in large projects. In this regard, IBM's Beehive project is groundbreaking. One can read in numerous research papers the ways in which Beehive has been implemented and tested on IBM employees, as researchers have documented basic user behaviors. Researchers have focused on an entire ESMP (Ehrlich, Lin, \& Griffiths-Fisher 2007) or on features such as tagging systems (Thom-Santelli, Muller, \& Millen 2008) and user profiles (Dugan et al. 2008). Beehive research papers often use a social capital perspective (Steinfield, DiMicco, Ellison, \& Lampe 2009) to establish links between ESMP uses, and connecting strategies constitute a recurring theme. Researchers have identified that IBM employees use Beehive as a platform to expand their professional networks, using it to communicate with colleagues across organizational levels (Wu, DiMicco, \& Millen 2010). IBM employees undertake a range of search and retrieving practices (Jennifer ThomSantelli, Millen, \& DiMicco 2010) and use Beehive as a knowledge repository (Thom-Santelli, Millen, \& Gergle 2011). Other case studies on ESMPs in organizations other than IBM exist, but have yielded limited insights. They show that employees use ESMPs to streamline their online behavior to work practices and organizational affiliation (Zhang, Qu, Cody, \& Wu 2010). Researchers have examined the challenges of adopting an organizational ESMPs. It is not uncommon to come across findings that highlight how employees continue to prefer to communicate via e-mail and chat software, and silently monitor news streams (Lüders 2013; Pettersen 2014. 2016). Consequently, one finds a pattern that a core group adopts SNS and maintains network activities, while a larger group uses 'older' forms of ICTs.

Therefore, the research horizon described appears limited and somewhat inchoate. Scholars have predominantly focused on the material properties of ESMPs and have contributed through 


\section{N}

NORDIC JOURNAL

of Science and Technology Studies

experimental theorizing. Absent from the research literature is the specific role that interpretation and practice take in employees' recursive use, with ESMPs a crucial aspect of work processes. Moreover, it appears that the research field has yet to adequately frame whether sharing can take on a different role than that intended, and what it means when technology is used differently in an organizational setting. This means that the research field can advance a research lens focusing on situations and enacting with emergent properties that come from the use of recursive technology, hence placing clearer emphasis on what people do with an ESMP. Thus, one can use a practice perspective on technology (Orlikowski 2000). A practice lens on ESMPs can also be used to fill a knowledge gap regarding the formation of knowledge-sharing processes in the use of ESMPs, facilitating our understanding of the unintended consequences of technology use in organizations. Here, Orlikowski and Gash's (1994) technological frames can be of assistance. Technological frames are defined as: "that subset of members' organizational frames that concern the assumptions, expectations, and knowledge they use to understand technology in organizations. This includes not only the nature and role of the technology itself, but the specific conditions, applications, and consequences of that technology in particular contexts" (Orlikowski \& Gash 1994, 178). The concept can enable us to frame how individuals and social groups in organizations alike make sense of a technology to determine their actions, allowing us to move beyond conceptualizing a technology's mere value and perception among users. Technological frames problematize the 'taken for granted' notions of a technology and can facilitate an understanding of how individuals and groups in organizations develop particular assumptions, expectations and knowledge of a new technology in an organizational setting. Orlikowski and Gash (1994) have illustrated technological frames in their study of the implementation of the groupware Notes in a consultant company. The researchers interviewed the implementers and adopters, grouping them into 'technologists' and 'users.' 'Technologists' was used to refer to technology staff, whereas 'users' comprised the organization's consultants. Orlikowski and Gash demonstrated a large set of differences in terms of expectations and actions, which they attributed to differences in technological frames. For example, the technologists viewed Notes as an enabler for information sharing, electronic communication, document management, and online discussion, which they believed could contribute to collaboration. The users' interpretation was different, viewing Notes' electronic e-mail features as a potential substitute for existing communication technologies such as fax and telephone. The technologists therefore framed Notes as a collaborative technology, whereas the users used it as a means for individual and personal communications.

\section{About the case and methods}

Norway is divided into nineteen large administrative units, called counties, and roughly 350 municipalities. Each county is governed by a County Authority (CA), rendering this form of governance the first form of subdivision in the country. The CA where the data for this case study were collected consists of a political structure, an administrative body, and welfare units. The political structure is an elected body consisting of the County Council, the County Executive Board, the County Principal Standing Committees, and the County Mayor. The County Council is supported by an administrative body, the County Administration, which implements and administers policies. The County Administration is organized into eight administrative units and an executive secretariat board. Other welfare units also exist, which play a role for citizens and produce services. Among others, these consist of high schools, libraries, dental services, and transportation. A large body of the CA workforce includes high school teachers, and in total the CA contains approximately 2,800 employees.

The study made use of an explorative qualitative research strategy. This approach was used to facilitate an in-depth investigation of the ways in which public employees working predominantly in the County Administration used the ESMP and interpreted sharing in an organizational setting. The study is primarily based

on qualitative research interviews. Written documentation was collected, but is not used as part of this paper and is thus excluded from the data analysis. However, the research design started with an informal approach to employees in the CA who had been responsible for the public procurement and implementation of the ESMP. They agreed to be part of the explorative qualitative study and recruited the informants. Eight informants were recruited, and they worked in different departments and holding positions, predominantly as advisors in the County Administration. The criterion for selection was that they were all users of or involved in the implementation of ESMP. In sum, eight semi-structured qualitative interviews with the use of a guide were completed. The interviews were undertaken one-to-one, meaning that only the researcher and the informant were present in the interview setting. Each interview lasted approximately one hour and focused on two main themes related to sharing: previous user sharing experience on social media, and how the individual used the ESMP to organize the sharing of work. Each interview was recorded using a digital audio recorder. The data were collected over two periods, from August to September 2013 and in February 2014. The study was based on informed consent and the informants were anonymized. The background of the informants is displayed in Table 1. 


\section{TABLE $]$}

\begin{tabular}{|c|c|c|c|c|}
\hline Gender & Number & Position & Duration & Date \\
\hline $\mathrm{F}$ & $1-1$ & Advisor & 1 hour & 27 Aug, 2013 \\
\hline $\mathrm{F}$ & $1-2$ & Middle Manager & 1 hour & 30 Aug, 2013 \\
\hline $\mathrm{F}$ & $1-3$ & Advisor & 1 hour & 5 Sep, 2013 \\
\hline $\mathrm{F}$ & $1-4$ & Exe. Director & 1 hour & 12 Feb, 2014 \\
\hline $\mathrm{M}$ & $1-5$ & Advisor & 1 hour & 10 Feb, 2014 \\
\hline $\mathrm{M}$ & $1-6$ & Advisor & 1 hour & 17 Feb, 2014 \\
\hline $\mathrm{F}$ & $1-7$ & Consultant & 1 hour & 18 Feb, 2014 \\
\hline $\mathrm{M}$ & $1-8$ & Advisor & 1 hour & 18 Feb, 2014 \\
\hline
\end{tabular}

Table 1: The background of informants.

\section{Data analysis}

This section presents the data analysis and seeks to answer the research question addressed earlier in the paper: how does a group of employees in the CA interpret the meaning of sharing? The emphasis here is on breaking down this notion by presenting four emergent themes from the open coding of the interview
Once the interviews were completed, they were transcribed. The data analysis was inspired by an open coding strategy of the interview data. Here, the main focus was on finding emerging patterns, which consisted of grouping and comparing the informants' perceptions, user patterns, and experiences of sharing. The informants' answers were grouped into four broad themes. In order to offer the informants a voice, direct quotations are used in the data analysis.

\section{TABLE 2}

\begin{tabular}{|c|c|}
\hline Theme No. & Name of theme \\
\hline 1 & Sharing as a facilitator of organizational change \\
\hline 2 & Sharing as a trigger for self-censorship and risk-taking \\
\hline 3 & Sharing in separate digital ecosystems \\
\hline 4 & Sharing as an individual informing strategy \\
\hline
\end{tabular}

Table 2: Emergent themes from the data analysis.

data. Each theme represents an adoption of the new workplace principle of sharing, and shows how individuals develop particular assumptions, expectations, and knowledge about its definition. Performing such an analysis can facilitate a clearer understanding of the meaning of sharing. The four themes are displayed in Table 2.

\section{Theme 1: Sharing as a facilitator of organizational change}

The first theme pertains to expectations of sharing as a facilitator of organizational change and represents an approach whereby top managers want their employees to work in a different way. This interpretation comes as little surprise, because the CA's top management was the initiator behind the ESMP. Sharing represents a "problem-solver" and can produce organizational belonging in the face of internal forces that may contradict unity. Certain professions, such as teachers - a large profession - are assumed to identify with the high schools in which they work and with their professional identity, rather than with a feeling of belonging to the CA. However, the motivation for implementing the social ESMP was related to disentangling a common problem with which most organizations struggle to cope today: escaping the meeting culture, e-mail overload, and the use of too many forms of IT:

There was a need to create an ESMP that considered the fact that we worked with various work surfaces. You had to open each system one at a time, just to approve an invoice. Our challenge was also to escape the 'hell of e-mail'. (1-4)

The top management aimed to simplify employees' work surface. This was related to the fact that employees worked across several non-integrated ICTs and stored information in different places, making it challenging to create an overview. In response, the top management argued that a single site that could work as the central access point connecting all the employees was required. This would be realized by replacing the intranet with the ESMP. Therefore, a project group was created to work with various drafts of a new interface design, which would break an established work pattern in the CA. While the intranet was run as an internal website on which a group colleague wrote internal news stories, the new design suggested that the ESMP should be the main site opened by employees each day, with embedded sharing features and URL links to each internal IT system. In this way, the ESM would be the melting pot where everybody talked about work. Afterwards, an organizational discourse emphasizing the importance of a sharing culture emerged:

It was clear that we needed something that could enable us to work with the culture across [the organization], knowledge of each other's work. My responsibility has been to legitimize sharing in the management structure. Parallel to that, we made attempts to raise discussion about organizational culture and work processes internally. Should we establish a sharing culture, 
in the sense that people can easily participate in and reinforce each other's work, or take part in reports, or take part in other kinds of things, take part in the knowledge we have, this requires a culture where [people] actively participate. (I-4)

However, translating and making sense of sharing into a manageable practice proved challenging, as it surfaced as ambiguous:

It sounds very good. It has a great positivity to it when it's presented, but not so great when you try it out in practice. At an early stage, there was a positive feel. You didn't know exactly what it was. There was this belief that we should change the work culture. (I-5)

Later, this awareness amplified as the initiators realized that the employees rarely started a work process by beginning from scratch - by creating a document in which everyone can engage, for example - but instead viewed sharing as an informing practice of circulating ready-made documents. A recurring theme was how sharing was directly linked to previous publishing habits.
The employees were accustomed to an article format, meaning that postings had an 'internal story' label attached to them. Participation involved performing simple tasks, like writing status updates, following colleagues, and updating profiles. The ESMP was an information channel where information was pushed out, not a platform in which one engaged in two-way dialogue. Furthermore, the employees fulfilled activities that required little commitment, such as posting a profile picture, writing status updates, tagging competence, or uploading completed documents. Beyond these actions there was little evidence that the users aimed to participate in activities requiring the performance of reciprocal actions:

Ninety percent of the information posted on the ESMP is not something that we've published. It's made by the organization. It has become a place where items are shared. It's divided between heavy and light documents. People share when documents are finished. You don't see many examples where people collaborate on a document, which is part of a work process. We haven't gone any further in changing work culture and the ways we work. (1-5)

\section{Theme 2: Sharing as a trigger for self-censorship and risk-taking}

The second theme emerging from the data analysis is that sharing includes a high degree of self-censorship and is associated with risk-taking. This pattern was seen among employees who use the ESMP and who were not part of the actual implementation process, who work across different departments and who are affiliated further down in the management structure. However, an interesting pattern consists of the ways in which earlier and alternative private social media platforms shape perceptions of appropriate net behavior. For example, the informants registered on social media services that became mainstream in the 2000 s (the informants' use is displayed in Table 3). The data indicates that the informants had a strong passive and critical approach to participation. They saw the benefits of sharing, but demonstrated a 'reading and textbook' approach consisting of monitoring others' actions and only frugally sharing about themselves. This molded a view that sharing was seldom regarded as a two-way communicative process between two parties:

I don't share information about what I've eaten for dinner, what I do during my evenings. I share if it is appropriate and relevant, not just one of those private things. Sometimes I post a picture of a mountain summit on Facebook. I have a pretty high threshold that the summit should be a little more interesting for others to see. $(\mathrm{I}-2)$

Therefore, it is more accurate to maintain that communicative practices are based on being informed and to inform, which ignores how a goal is to engage with an equal to create knowledge. This

\section{TABLE 3}

\begin{tabular}{|c|c|c|c|c|c|c|c|}
\hline Gender & Number & Position & Facebook & Twitter & LinkedIn & Instagram & Google Drive \\
\hline $\mathrm{F}$ & $\mid-1$ & Advisor & $x$ & - & $x$ & - & - \\
\hline $\mathrm{F}$ & $1-2$ & Middle Manager & $x$ & $x$ & $x$ & $x$ & - \\
\hline $\mathrm{F}$ & $1-3$ & Advisor & $x$ & - & - & - & - \\
\hline $\mathrm{F}$ & $1-4$ & Exe. Director & $x$ & $x$ & $x$ & - & - \\
\hline M & $1-5$ & Advisor & $x$ & $x$ & $x$ & - & $x$ \\
\hline M & $1-6$ & Advisor & $x$ & - & - & - & - \\
\hline $\mathrm{F}$ & $1-7$ & Consultant & - & - & - & - & - \\
\hline$M$ & $1-8$ & Advisor & $x$ & $x$ & $x$ & - & $x$ \\
\hline
\end{tabular}

Table 3: The informants' use of social media platforms. 
is reflected in beliefs about socializing and the means of ascribing social media with personal labels. For example, Facebook belongs to the private sphere and is used for "scrolling after fun stuff and setting likes." LinkedIn is a "CV database," while Twitter is a medium where "one only sends URL links to news you have already read." Such beliefs hint at what is acceptable to share on which platform:

There are people who write things that shouldn't be shouted out loud. One gets the impression that, "I'm sad today". If anyone had a nice trip, which is worth writing about, then you can do it, by all means. It's easy to explain it, but when you see it, it gets difficult to say it with words. There are some things that are just a bit 'intimate', very personal stuff. It doesn't belong on social media, because it doesn't concern everybody. (I-6)

Grooming and gossiping are disregarded, and publishing of overly personal information evokes intimacy and is taboo. Instead, the informants had clear perceptions that what should be shared had to be interesting and relevant, meaning that the value of what can be shared has to be informative and of high quality. This creates boundaries delineating how one should engage on the ESMP, which are manifested in the form of distinctions. For example, the most common trait was to draw distinctions between work and non-work-related use, and external and internal use. Here, Twitter is work-related and is used because politicians interact in the Twittersphere, and it forms an arena for public debate. Some informants see Twitter as a "listening post" where one can monitor what is going on and to stay up-to-date with current events:

I use Twitter because it gives me something related to my work and because I follow public debates. So, I started paying attention to what was going on Twitter. In our department, we follow public debates. (I-8)

Consequently, one could expect that engaging on the ESMP should represent a challenge. Yet, the data suggest otherwise, as sharing on the ESMP was connected with risk-taking, expressed in the informants' views of their willingness to make a work process transparent to others. Here, the informants placed themselves on a scale from relatively open to very restricted in what they shared:

I try to set an example. When I create a document, I publish it right away. It says it's a document in progress, which we're working on. (I-2)

I don't have a problem with posting something that is not one hundred percent complete. I would have made it clear that this is 'work in progress' which I want feedback on. (I-5)

However, we also find examples illustrating the risk-taking associated with publishing work in progress. This is related to the idea that informants assume that they can be criticized, meaning that published and unfinished work can create misunderstandings:

One thing is that some us find it a bit uncomfortable to share things that are not finished, because then we get criticized. It becomes uncomfortable when it's not completed. If things are just published and not finished, it can cause more harm, because it creates sanctions on something that it was not intended to be. We have specific discussions within our work areas, documents concerning the management side and on the political aspects, which we publish. When things are at a certain stage, a working document, it is not intended that everybody should see it. If there are many who use it, they can abuse it in a number of contexts. (I-2)

The data also show that informants seek 'approval' from their closest manager to publish content on the ESMP. Rather than deciding independently, for example, as the basis for sharing a document, informants enforce a quality-safety practice where they ask permission from an authority in the management structure:

Things that are unfinished and not approved can create panic when it is a different figure from what you think is going to be on paper. If we begin to rewrite the CA's economy and everyone can read that, there will be something new to most people. Many people absorb it, even when it is wrong. It creates a lot of 'storm' in your organization if it is not correct. I can take an example from the corporate governance program, which has an indicator called 'financial statements and budgets', which shows how much of a deficit/surplus we have to date. It is an indicator that gets its numbers straight from our accounting system. When we updated the financial system, the indicator 'froze' itself in Corporater and showed figures from November 2013. This is a completely wrong figure. We have notified about that on the ESMP, but still I keep getting phone calls that the figures are wrong. (I-6)

Another informant gave a similar example:

If I am to work on a case, I want to have the final answer before I publish it. I can give you an example, which applies to the CA's dental clinics. When they want to send over a thing, in the process, things that go to debt collection arrive on my desk. I have not posted anything during the process because I wanted my manager to look at the draft along with other managers. It's the way that I work, the way I think: the routine should be completed before any dentist gets access. Considering that you want to have a unified management involved, they have to see the final result first. Then time passes, and we have a routine: we end up with draft $C$ and $D$, until we finally land on something. (1-7) 


\section{Theme 3: Sharing in separate digital ecosystems}

The third theme from the interview data illustrates the ways in which informants adopt social media services and construct knowledge-sharing processes that form part of a work process. Thus, it makes sense to state that the informants create separate digital ecosystems that are used when they perceive that the ordinary ICTS provided by the CA are insufficient to performing their work, which influences employees to look for alternatives, a technology-adoption that occurs 'under the radar' of the IT department.

Looking at particular practices, an informant explained how they combined Dropbox and Google Drive as part of a work process that was used to complete the organization of a public procurement. In several cases, the CA works together with the neighboring municipalities. As part of the process, the CA assumes the lead role as the public buyer and lead organizer, meaning that the $C A$ acts on behalf of many municipalities to achieve greater benefits for all. This work requires collaboration with colleagues in other municipalities. In that regard, one can expect that colleagues in different municipalities have diverse needs and competencies, hence many persons voice different opinions and needs. This will lead to long e-mail exchanges and numerous attached documents, with the effect that one quickly loses the overview. Instead of sending back and forth large numbers of e-mails with large documents attached, the respondents used Dropbox or Google Drive to increase the efficiency and economy of the work process for everybody:

We created a Dropbox account because we don't have the same e-mail system or share the same case management system. And it's challenging. You don't get Dropbox solutions on the PCs here. The IT department thinks it's unsecure, [lacking] information security. We need tools to do our job, so we ended up defying that a bit and we downloaded the software to our PCs. Sometimes it happens that we use Google Drive when working with external partners. I used Google Drive to share documents more efficiently than by e-mail, before they get too large. (I-8)
Another practice is how Facebook groups are used either as information repositories or for external communication with particular groups who use the welfare services provided by the CA. Here, one does not find examples of practices that demonstrate knowledge sharing between several parties, but merely how Facebook groups are used as public bulletin boards, where online sharing again represents an informing practice. For instance, an employee was a representative in one of the CA's worker unions and interacted with representatives from other CAs. In the process, they created a Facebook group that enabled them to stay in contact and inform one another:

It was part of a different role, which was part of a task I had here in the CA. I had contact with others with the same role in other CAs. We used the Facebook group to share information that was more or less of the same nature. It was a way to share knowledge on issues of health and safety at work. (I-1)

Another public employee explained how they created a Facebook group to communicate with a group of citizens the CA serves directly, students in high schools. The Facebook group was created on the assumption that students would contact the CA there; given that students are in the social media landscape, they concluded that the CA also needed to be present in a similar capacity. After some years of use, the Facebook group has roughly 300 'likers,' but expectations have not turned into reality. Relatively few requests from students have been seen. Instead, it has turned into more of a public bulletin board where information is posted:

It runs every day. We don't get many requests. We publish when we have specific information. We were unsure whether it would be an active user channel. I think it's going to become that in the long run. We intend to continue to use it and improve its uses, and even get more users. (I-3)

\section{Theme 4: Sharing practice as an individual informing strategy}

The fourth theme emerging from the analysis demonstrates how the employees turn sharing into individual informing strategies, which arguably fulfill a goal of complying with an overall objective to share on the ESMP. The fourth theme additionally demonstrates the challenges associated with performing sharing in an internal organizational setting, and translation problems connected with practicing sharing as a two-way communication act, given that it again becomes an informing and pushing strategy of camera-made information to an audience that does not respond. This is illustrated by scrutinizing a particular feature deemed as being important for creating the conditions for sharing on the ESMP, so-called 'rooms.' Rooms can best be described as Facebook groups or information repositories that operate as spaces for cooperation. Within them, users can upload and download documents, follow people, and receive messages about recent activities. The rooms have members and were grouped according to the CA's department structure and across departmental borders.

In considering particular overall user experiences, the data show that the informants adopted the rooms. They registered members and followed rooms, uploaded documents and so forth, such that it was common to follow between two and five rooms. Afterwards, 


\section{$\$$}

NORDIC JOURNAL

of Science and Technology Studies

the informants experienced challenges, illustrating the disadvantages and benefits associated with sharing. First, the informants created rooms and registered members who worked in the same department or in the same field as themselves. Second, the findings indicate that upload documents consisted of re-published information that was already stored in other places. A lack of data exists that shows that employees created new documents and began to co-write them in real-time; rather, they uploaded approved and ready-made documents that were only read for notification purposes. This indicates that sharing is an informing practice to a large audience, which does not invite a two-way communication process. Third, all informants reported that little interaction (such as participation and reading discussions) occurred in the rooms. In sum, users framed the rooms as information repositories rather than as sites for collaboration. Thus, we see the pattern that employees with super-user status - users who enjoyed an administrative role in the rooms - tried to stimulate increased engagement, which represented an outcome of a lack of responses to their informing practices. In order to reach the top management goal of sharing, super-users adopted particular roles and strategies to promote participation, which in turn illustrates the challenges pertaining to sharing.

In reviewing these practices, it can be noted that one super-user would adopt an 'online gardener' strategy and attempt to encourage co-workers to engage in the rooms she administrated. This is not dissimilar to an automated e-mail notification feature, which is generated following a period of interactivity in a knowledge repository. She assumed the role of a sharer and pusher of information, which consisted of sending friendly e-mail reminders when she uploaded new documents:

I send an e-mail to everyone who has an interest in the room and then I share information with them that it's posted on the ESMP. Then I invite them to follow the room, because there is information there that is relevant to them. I say that it will only be posted there. That I've done for about a year. (I-1)

This means informing across multiple channels, turning sharing into a practice of double in-forming. Afterwards, the user questioned the value of sharing and was uncertain about the extent to which her efforts were worthwhile, a thought shared by another informant:

I note that there are not many who follow the rooms, after many invitations and reminders to others who I think might have an interest in it. And the thoughts come. Do we spend unnecessary time on posting information that people do not read anyway? (I-2)

This raises the question of whether the room members post material and use the rooms as intended. For example, after uploading, an informant also received phone calls to ask if the same documents could be sent by e-mail:
I often get the question, if I can send them an e-mail when there is new information in the rooms. We have decided on that - no, we don't send an extra e-mail. We put it out there, and then people must seek it out themselves. I feel that people don't pay attention to all that is posted in the rooms. They would have paid attention if we had sent it in an e-mail. But we have made a conscious choice on that. I think that people read it if they get an e-mail because it's a direct message aimed at them, rather than having to search for the information themselves. (I-2)

This experience shows the start of a disengagement regarding sharing, as it vanishes and becomes overtaken by other assignments whose completion is deemed more important:

We have two rooms. I post a lot of information in them. I try to ensure that new information is posted. But I do not use the opportunity to follow other rooms on the ESMP, for example, as I had hoped and thought I would. It disappears into my daily work life. When I need information, I don't find it with the search mechanisms that we have today as we had with the intranet, although there is more information out there now. But now, I think it is harder to find information. (I-2)

Informing over a long period of time creates an awareness that attached to sharing is an embedded information overload problem. This is illustrated by repeatedly performing an informing practice wherein users redirect information that is stored elsewhere. For example, while information is stored locally on hardware or in a local folder, such information is exposed and redistributed multiple times in the rooms. Hence, making information available to create transparency led to other consequences:

The intention with the ESMP was that we should move away from local storage of information in our own local folder structures. Everything was to be stored on the ESMP. I'm skeptical of it, because it is such a vast amount of information that it makes it difficult to identify what is relevant. We end up with huge hits when we search, and we spend a lot of time on finding out what is relevant. And when we do not have the rigid old structure, which we had under the intranet, we spend a lot of time looking among all the hits we get. I think we would have wanted it to be a little stricter on what should be stored. Things should be deleted, if they are considered [ir]relevant. I'm also skeptical that we use the ESMP as a primary storage source for everything. I'm also concerned because we could forget the formal filing and procedural rules that we have to deal with. When we publish on the ESMP, we think we preserve it forever, and that's not right. There are some formal things that make me skeptical. The most concerning thing, however, is that it has become such a huge volume of documentation. And when it comes to relevant and non-relevant information on individual characteristics, I am a bit skeptical of that Facebook style of writing status updates. I think it's nice to have colleagues, but it's more interesting knowing how we are 
professionally connected. We are a large organization. If all of us post information that we were sick, and that we are looking forward to the weekend, there is a limit to how much of that information I want to see. I think it takes another turn and we're moving towards that side. That part I'm not thrilled over. I'm one of those who think that when I'm at work, I'm at work. People can tell me interesting things that are useful or fun for me to learn at work. I don't want a lot of private information, which I'm not related to. (I-2)

Exposure to excessive information instigates users to enforce a personal filter mechanism and to return to old work habits such as using e-mail. This results in the creation of distance from knowledge formation processes and the prevalence of disengaged users:

In the start, when it was brand new, I tried to make use of any opportunity, which was not in the intranet. We had the possibility to create rooms. I did that and invited people. I joined other rooms. But, afterwards, I failed to follow up all that. In neither of the rooms I administer now, did I manage to develop anything. I'm rarely there and don't check the rooms that I am a member of. (I-3)

This user saw the rooms as an opportunity to improve conditions for interaction with the high schools with which she has frequent contact as part of her work. Much of the daily contact consists of sending general information. Instead of sending all of this information via e-mail, it could be transferred to the rooms, but she did not manage to uphold the goal of sharing:

I haven't had time to prioritize the room. They come far down on my priority list. My workday is packed with to-do tasks. To sit down to try to use the possibilities and communicate in the rooms has instead led me not doing that. Now, I don't bother checking notifications from the rooms I administer or follow or what my colleagues have written in their status updates. I skip that very fast and I go directly to check my e-mails. (I-3)

This pattern of disengagement was seen in another experience. An informant explained that the challenges of generating engagement were related to aspects of the user interface itself. For example, it was difficult to ascertain whether the rooms were used by others as no panel to show numbers of visitors existed. The male user argued that the information shared in the rooms was already available and ready-made in other spaces, which meant that it was stored by co-workers in their e-mail inboxes. However, other matters drew attention:

The challenge with the ESMP is that there are too many rooms. It's almost like we have a room for each employee. You have to click on a link to get to somewhere. And then you have to go back again and click on a new link again, so it will be many rounds, just to get hold of the information you're looking for. $(I-6)$

Although a number of the informants were uncertain as to the extent to which their sharing in the rooms was of benefit, another user shared a quite different opinion. A female user working with accounting explained that the rooms represent a type of 'manual.' She was an active user and saw the benefit of retrieving and finding information that had been shared by others.

For example, I'm working in the accounting system and I find out that I need to get hold of a manual or retrieve information on an account. I go on the ESMP. There, I locate documents or things that are written about the case I'm working on. I'm a member of all the rooms that have something to do with accounting, a factor allowing me to know what we've posted and what others ask about. (I-7)

The rooms are beneficial in different ways. For example, they are information depositories, where one can find quick answers, as they narrow down the need for searching. Alternatively, she would have to search for the same information in larger web-based databases.

Since they exist, they are easy available. They are part of a knowledge you can easily use. They are there if you need to be reminded about something. For example, in accounting, there are clear definitions, clear rules for use; there's a clear date of notice for certain things. Things that are not so relevant one day, I often get information about in advance. But then I get questions from colleagues working in other departments, who ask about a deadline. What date is set as a deadline for the final reporting? Now, I know where I can quickly get and give an answer back on that. It's not necessarily that I have that knowledge in my head, but now I have good knowledge of where the answer is located. (I-7)

\section{Discussion}

Orlikowski and Gash (1994) utilized a practice and interpretation perspective to conjecture that Notes can be interpreted in diverse ways, revealing differences in intent and actual use. Their analysis highlighted the notion that implementers see Notes as a tool for organizational change and collaboration, whereas end-users

interpret it as a means of individual and personal communication. Using a similar research lens with alternative empirical material - the implementation of an ESMP in a Norwegian public organization - what clues are provided that help to answer the paper's research question? 
The most important finding is the contradictoriness of a top management initiative intended at simplifying employees' work surface in a public organization that seems to have had the opposite outcome in terms of end-users' use and action. Sharing, introduced as a new workplace principle, was expected to create transparency and enhance the flow of internal communication, but when the end-users attempted to translate sharing into a manageable practice - as the basis for participation in a knowledge formation process - they interpreted sharing as a complicating work practice, with the larger consequence of producing disengaged users. This is primarily related to the fact that users are not performing a sharing practice, that is, a two-way communication process whereby knowledge is created through collaboration. Rather, the data analysis shows that the users engaged in informing practices to fulfill the goal of sharing, an aspect that has been demonstrated throughout the data analysis section. This informing practice - which represents an essential ingredient in creating a knowledge-sharing process - is performed on the premise of informing an audience and of being informed. Moreover, the informing practice is seldom the start of a knowledge process where two users exchange information to create knowledge by reflection on action, for example. Instead, sharing is carried out by re-publishing ready-made and approved official documents found elsewhere in the CA, creating an unmanageable information overload problem that encapsulates the challenges in forming a sensible knowledge-sharing process in practice. Furthermore, clues are provided regarding what is actually shared, which in this explorative case study pertains to information that is already known. Sharing proves to be problematic and is associated with risk-taking for those involved, leading to the enforcement of self-censorship and the construction of separate and private workplaces that the informants deem beneficial to completing their work. In contrast, the users institute personal filters and return to a work surface that they believe works, which in most cases is e-mail. In other words, sharing in this case study is interpreted and performed as informing or as being informed.

\section{Conclusion}

The main outcomes of this explorative case study have been an examination of the term 'sharing,' and demonstrating the challenges involved in introducing it as a workplace principle in a public organization. Moreover, when public employees attempt to perform and make sense of 'sharing' in practice, a two-way communication

practice emerges that can be misidentified and performed as a practice based on informing an audience and of being informed, hence causing an information overload problem and the prevalence of disengaged users in organizational life.

\section{References}

Ardichvili, A. 2008. Learning and knowledge sharing in virtual communities of practice: Motivators, barriers, and enablers. Advances in Developing Human Resources 10 (4): 541-554.

Ardichvili, A., Maurer, M., Li, W., Went Ling, T., \& Stuedemann, R. 2006. Cultural influencers wed knowledge sharing through online communities of practice. Journal of Knowledge Management 10: 94-107.

Ardichvili, A., Page, V., \& Wentling, T. 2003. Motivation and barriers to participation in virtual knowledge sharing communities of practice. Journal of Knowledge Management 7 (1): 64-77.

Callon, M. 1986. Some elements of a sociology of translation: Domestication of the scallops and the fishermen of St Brieuc Bay. In Power, action and belief: A new sociology of knowledge, edited by J. Law. London: Routledge \& Kegan Paul.

Chen, C. J., \& Hung, S. W. 2010. To give or to receive? Factors influencing members' knowledge sharing and community promotion in professional virtual communities. Information and Management 47 (4): 226-236.

Dugan, C., Geyer, W., Muller, M., DiMicco, J., Brownholtz, B., \& Millen, D. R. 2008. It's all 'about you': Diversity in online profiles. Proceedings of the 2008 Conference on Computer Supported Cooperative Work, 703-706.

Ehrlich, K., Lin, C.-Y., \& Griffiths-Fisher, V. 2007. Searching for experts in the enterprise: Combining text and social network analysis. Proceedings of the 2007 international ACM conference on Supporting group work, 117-126.

Ellison, N. B., Gibbs, J. L., \& Weber, M. S. 2015. The use of enterprise social network sites for knowledge sharing in distributed organizations: The role of organizational affordances. American Behavioral Scientist 59 (1): 103-123.

Fulk, J., \& Yuan, Y. C. 2013. Location, motivation, and social capitalization via enterprise social networking. Journal of ComputerMediated Communication 19(1): 20-37.

Gibbs, J. L., Rozaidi, N. A., \& Eisenberg, J. 2013. Overcoming the "ideology of openness": Probing the affordances of social media for organizational knowledge sharing. Journal of ComputerMediated Communication 19(1): 102-120.

Gibson, J. J. 1986. The ecological approach to visual perception. Mahwah, NJ: Erlbaum

Kaplan, A. M., \& Haenlein, M. (2010). Users of the world, unite! The challenges and opportunities of Social Media. Business Horizons, 53(1), 59-68.

Kosonen, M. 2009. Knowledge sharing in virtual communities - A review of the empirical research. International journal of Web 
Based Communities 5(2): 144-163.

Latour, B. 1987. Science in action: How to follow scientists and engineers through society. Milton Keynes: Open University Press.

Latour, B. 2005. Reassembling the social: An introduction to actor-network-theory. New York: Oxford University Press.

Leonardi, P. M. 2013. Theoretical foundations for the study of sociomateriality. Information and Organization 23 (2): 59-76.

Leonardi, P. M. 2014. Social media, knowledge sharing, and innovation: Toward a theory of communication visibility. Information Systems Research 25 (4): 796-816.

Leonardi, P. M. 2015. Ambient awareness and knowledge acquisition: Using social media to learn "Who knows what" and "Who knows whom". Management Information Systems Quarterly 39 (4): $747-776$.

Leonardi, P. M., \& Barley, S. R. 2010. What's under construction here? Social action, materiality, and power in constructivist studies of technology and organizing. The Academy of Management Annals 4 (1): 1-51.

Leonardi, P. M., Huysman, M., \& Steinfield, C. 2013. Enterprise social media: Definition, history, and prospects for the study of social technologies in organizations. Journal of Computer-Mediated Communication 19 (1): 1-19.

Leonardi, P. M., \& Meyer, S. R. 2015. Social media as social lubricant: How ambient awareness eases knowledge transfer. American Behavioral Scientist 59 (1): 10-34.

Lüders, M. 2013. Networking and notworking in social intranets: User archetypes and participatory divides. First Monday 18(8).

Majchrzak, A., Faraj, S., Kane, G. C., \& Azad, B. 2013. The contradictory influence of social media affordances on online communal knowledge sharing. Journal of Computer-Mediated Communication 19 (1): 38-55.

McAfee, A. 2006. Enterprise 2.0: New collaborative tools for your organization's toughest challenges. Available from http://andrewmcafee.org/2006/og/enterprise_2O_inclusionists_and_ deletionists/

Merton, R. K. 1936. The unanticipated consequences of purposive social action. American Sociological Review 1 (6): 894-904.

Mutch, A. 2013. Sociomateriality - Taking the wrong turning? Information and Organization 23 (1): 28-40.

Oostervink, N., Agterberg, M., \& Huysman, M. 2016. Knowledge sharing on enterprise social media: Practices to cope with institutional complexity. Journal of Computer-Mediated Communication 21 (2): $156-176$

Orlikowski, W. J. 2000. Using technology and constituting structures: A practice lens for studying technology in organizations. Organization Science 11 (4): 404-428.

Orlikowski, W. J. 2007. Sociomaterial practices: Exploring technology at work. Organization Studies 28: 1435-1448.

Orlikowski, W. J. 2010. The sociomateriality of organisational life:
Considering technology in management research. Cambridge Journal of Economics 34 (1): 125-141.

Orlikowski, W. J., \& Gash, D. C. 1994. Technological frames: Making sense of information technology in organizations. Acm Transactions on Information Systems 12 (2): 174-207.

Orlikowski, W. J., \& Scott, S. V. 2014. What happens when evaluation goes online? Exploring apparatuses of valuation in the travel sector. Organization Science 25 (3): 868-891.

Orlikowski, W. J., \& Scott, S. V. 2008. Sociomateriality: Challenging the separation of technology, work and organization. The Academy of Management Annals 2 (1): 433-474.

Pettersen, L. 2014. From massproduction to masscollaboration: Institutionalized hindrances to social platforms in the workplace. Nordic Journal of Science and Technology 2 (2): 29-40.

Pettersen, L. 2016. The role of offline places for communication and social interaction in online and virtual spaces in the multinational workplace. Nordicom Review 37(Special Issue): 131-146.

Steinfield, C., DiMicco, J. M., Ellison, N. B., \& Lampe, C. 2009. Bowling online: Social networking and social capital within the organization. Proceedings of the Fourth International Conference on Communities and Technologies, 245-254.

Suchman, L. A. 2007. Human-machine reconfigurations: Plans and situated actions. Cambridge, UK: Cambridge University Press.

Thom-Santelli, J., Millen, D. R., \& DiMicco, J. M. 2010. Characterizing global participation in an enterprise SNS. ICIC'10: 251-254.

Thom-Santelli, J., Millen, D. R., \& Gergle, D. 2011. Organizational acculturation and social networking. Paper presented at the Proceedings of the 2011 Conference on Computer Supported Cooperative.

Thom-Santelli, J., Muller, M. J., \& Millen, D. R. 2008. Social tagging roles: Publishers,evangelists, leaders. Proceeding of the TwentySixth Annual SIGCHI Conference on Human Factors in Computing Systems, 1041-1044.

Treem, J. W., \& Leonardi, P. M. 2012. Social media use in organizations: Exploring the affordances of visibility, editability, persistence, and association. Communication Yearbook 36: 143-189.

Vaast, E., \& Kaganer, E. (2013). Social media affordances and governance in the workplace: An examination of organizational policies. Journal of Computer-Mediated Communication 19 (1): 78-101.

Wasko, M. M., \& Faraj, S. 2005. Why should I share? Examining social capital and knowledge contribution in electronic networks of practice. Mis Quarterly 29 (1): 35-57.

Wu, A., DiMicco, J. M., \& Millen, D. R. 2010. Detecting professional versus personal closeness using an enterprise social network site. The 28th International Conference on Human Factors in Computing Systems, 1955-1964.

Zhang, J., Qu, Y., Cody, J., \& Wu, Y. 2010. A case study of micro-blogging in the enterprise: Use, value, and related issues. Atlanta, GA. 